\title{
Vías de señalización anabólicas en el hueso y su potencial aplicación en la terapéutica
}

\author{
Ariana Sierra ${ }^{1}$, Adriana Medina ${ }^{2}$, William Rojas ${ }^{3}$, Henry Tovar ${ }^{4}$, Carlos Révérend ${ }^{5}$, \\ Alfonso Suárez $z^{6}$
}

\author{
${ }^{1}$ Residente de Endocrinología II año. FUCS- Hospital San José. \\ ${ }^{2}$ Instructor Asistente, Servicio de Endocrinología. \\ FUCS- Hospital San José. \\ ${ }^{3}$ Profesor Asociado, Servicio de Endocrinología. \\ FUCS- Hospital San José. \\ ${ }^{4}$ Instructor Asociado, Servicio de Endocrinología. \\ FUCS- Hospital San José. \\ ${ }^{5}$ Biólogo. Maestría en Bioquímica. Profesor de Ciencias básicas \\ Facultad de Medicina FUCS. \\ ${ }^{6}$ Médico. Maestría en Genética Médica. Profesor Asistente de la \\ Facultad de Medicina de la FUCS.
}

\section{Resumen}

$\mathrm{P}$ ara el manejo actual de la osteoporosis contamos con la terapia antirresortiva, que estabiliza la arquitectura ósea sin lograr su restauración y la anabólica (teriparatida: único aprobado por la FDA) que restaura y aumenta la masa ósea. La identificación de reguladores moleculares con efecto anabólico sobre el hueso ha permitido el desarrollo de nuevas terapias para el manejo de esta patología cada vez más prevalente.

La vía de señalización $W n t / \beta$-catenina aumenta la masa ósea a través de la diferenciación de células mesenquimales hacia osteoblastos y mediante el estímulo de la replicación de preosteoblastos e inhibición de la apoptosis de osteoblastos y osteocitos, siendo las proteínas esclerostina y DKK1 (Dickkopf 1) sus principales antagonistas. Se encuentran actualmente en desarrollo anticuerpos monoclonales humanizados contra estas proteínas (Ac anti esclerostina y anti DDK1) que tienen a un efecto formador de hueso.

Otra alternativa de uso local es la Proteína Morfogénica de Hueso 2, recombinante humana (rhBMP-2), con capacidad osteogénica, que ha demostrado aumentar la resistencia ósea en zonas de fracturas, acelerando la consolidación de las mismas.

Estos nuevos reguladores del remodelado óseo representan una alternativa terapéutica de la osteoporosis y otros trastornos asociados al desequilibrio entre la resorción y la formación ósea.

Palabras clave: osteoporosis, esclerostina, DKK1, anabólico óseo.

\section{Summary}

The current management of osteoporosis includes antiresorptive therapy, which stabilizes bone architecture without achieving its restoration, and anabolic therapy (Teriparatide: the only agent approved thus far by the FDA), which restores and increases bone mass. The identification of molecular regulators with anabolic effect on bone has allowed for developing new therapies for the management of this increasingly prevalent condition.

The Wnt/ß-catenin signaling pathway increases bone mass via differentiation of mesenchymal cells into osteoblasts, stimulation of pre-osteoblasts replication and inhibition of the apoptosis of osteoblasts and osteocytes, with the proteins Sclerostin and DKK1 (Dickkopf 1) being its main antagonists. Humanized monoclonal antibodies against these proteins (anti-sclerostin and anti-DDK1 Ab), which have bone forming effects, are currently being developed.

Another alternative is the local use of human recombinant bone morphogenetic protein 2, (rhBMP-2), a protein with osteogenic capacity, which has been shown to increase bone strength at fracture areas, accelerating their consolidation.

These new bone remodeling regulators represent a therapeutic alternative for osteoporosis and other disorders associated with an imbalance between bone resorption and formation.

Key words: Osteoporosis, Sclerostin, DKK1, osteo-anabolic.

\section{Introducción}

La remodelación ósea llevada a cabo en las unidades básicas multicelulares es necesaria en el esqueleto normal para el mantenimiento de la homeostasis del calcio, la reparación de microlesiones, la adaptación a cargas mecánicas y la eliminación del tejido envejecido ${ }^{(1,2)}$. Ésta juega un papel importante en el mantenimiento de la masa y estructura ósea durante la edad adulta y su equilibrio asegura una DMO (densidad mineral ósea) estable, por tanto el incremento de la resorción o la disminución de la formación del hueso conduce a pérdida ósea y osteoporosisis).

Teniendo en cuenta que la incidencia de fracturas osteoporóticas aumenta con la edad y su prevalencia cada vez es mayor, se requiere la aplicación de medidas destinadas a re- 
ducir su riesgo y a asegurar un adecuado manejo a través de nuevas terapias ${ }^{(4)}$. Actualmente contamos con dos estrategias para el tratamiento de la osteoporosis, la terapia antirresortiva y la terapia anabólica. Los antirresortivos, cuya célula diana son los osteoclastos, inhiben su efecto estabilizando la arquitectura ósea sin lograr su restauración. La otra alternativa es la terapia formadora de hueso o anabólica, cuyo blanco son los osteoblastos, permite la restauración y aumento de la masa ósea, promoviendo la diferenciación osteoblástica a partir de células mesenquimales. Hasta el momento, el único tratamiento aprobado por la FDA para este fin es la PTHr (teriparatida) ${ }^{(2,5)}$.

Los avances en la comprensión de los reguladores moleculares de la remodelación ósea han llevado a la identificación de nuevas dianas terapéuticas. Estas nuevas moléculas con efecto anabólico sobre el hueso promueven señales que aumentan el número de células osteoblásticas, permitiendo la replicación o diferenciación de preosteoblastos a osteoblastos o disminuyendo la apoptosis de las células maduras. Contamos con dos vías de señalización con estas características, la Wnt $\beta$-catenina (Wingless tail/ $\beta$-catenin) y la BMP (bone morphogenetic protein), cuyos estímulos se constituyen como nuevas alternativas formadoras de hueso ${ }^{(2,6)}$.

\section{Diferenciación de las células mesenquimales}

Los precursores mesenquimales expresan factores de transcripción que permiten su diferenciación hacia adipocitos, osteoblastos o condrocitos. Las vías anabólicas promueven el desequilibrio de la expresión de estos factores a favor de la diferenciación osteoblástica, permitiendo la expresión de Runx2 (runt-related transcription factor 2), Osx (osterix), ATF4 (Activating transcription factor 4), Msx2 (Msh homeobox 2), claves para adquisición del fenotipo osteoblástico (figura 1) ${ }^{(7,8)}$.

\section{Señales que determinan el destino y la función de las células osteoblásticas}

\section{Vía de señalización Wnt / $\beta$-catenina}

Wnts (Wingless tail) o ligandos Wnt constituyen una familia de glicoproteínas secretadas (factores de crecimiento), ricas en cisteína. En mamíferos existen 19 tipos. Sus funciones son: regulación del crecimiento, diferenciación, función y muerte celular, durante el desarrollo embrionario y en la vida adulta $^{(9,10)}$.

Las vías activadas por Wnt aumentan la masa ósea a través del compromiso de la célula mesenquimal hacia su diferenciación osteoblástica, la estimulación de la replicación de preosteoblastos y la inhibición de la apoptosis de osteoblastos y osteocitos. En los osteoblastos, la señalización Wnt se lleva a cabo a través de la unión a receptores de membrana de la familia Frizzled (Fz) y correceptores LRP5/6 (low-density lipoprotein receptor-related protein 5 and 6 (LRP5/6) coreceptors), inhibiendo la actividad de GSK3 (glycogen synthase kinase 3), evitando así la degradación de su sustrato, la $\beta$-catenina. Ésta se acumula en el citosol y se transloca al núcleo, donde actúa como un coactivador transcripcional LEF/TCF (factor linfoide de unión al promotor de células T) (figura 2) ${ }^{(7,8,9)}$.

Si no se expresa Wnt o si su unión a los receptores se inhibe, la degradación de $\beta$-catenina es facilitada a través de un complejo de degradación formado por las proteínas APC (Adenomatous polyposis coli), Axin y GSK3. Esta última fosforila a $\beta$-catenina, para que sea degradada por la vía ubiquitina/ proteasoma $\beta$-TrCP $(\beta$-TrCP-mediated ubiquitin/proteosome pathway) (figura $\mathbf{3})^{(7,8,9)}$.

\section{Proteínas esclerostina y DKK1: principales antagonistas de la señalización Wnt}

\section{Figura 1. Diferenciación de las células mesenquimales}

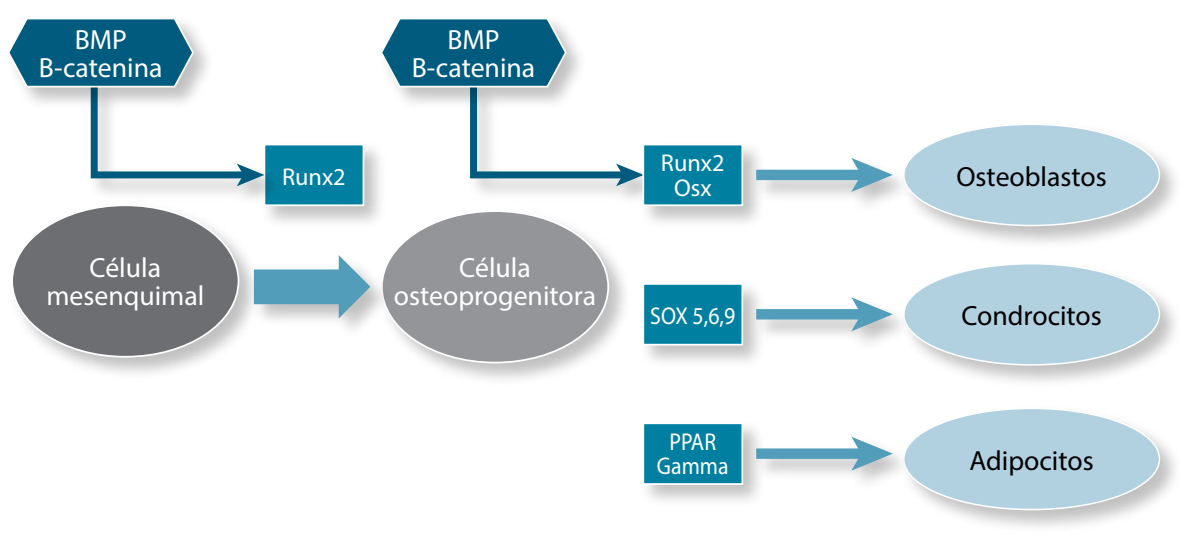

Modificado de Archives of Biochemistry and Biophysics 2008; 473:98-105.

\section{Esclerostina}

Es una glicoproteína de 190 aminoácidos, miembro de la familia de proteínas DAN/Cerberus. Su producción es regulada por el gen SOST situado en el cromosoma $17 q^{(11)}$. La expresión de esclerostina está relativamente restringida al esqueleto adulto, es principalmente producida por osteocitos, condrocitos articulares y débilmente por otros órganos como el riñón ${ }^{(12)}$.

Esclerostina se une al correceptor LRP5/6, inhibiendo de este modo la señalización Wnt/ $\beta$ catenina, reduciendo la formación 
Figura 2. Vía de señalización Wnt/ $\beta$-catenina: vía activa

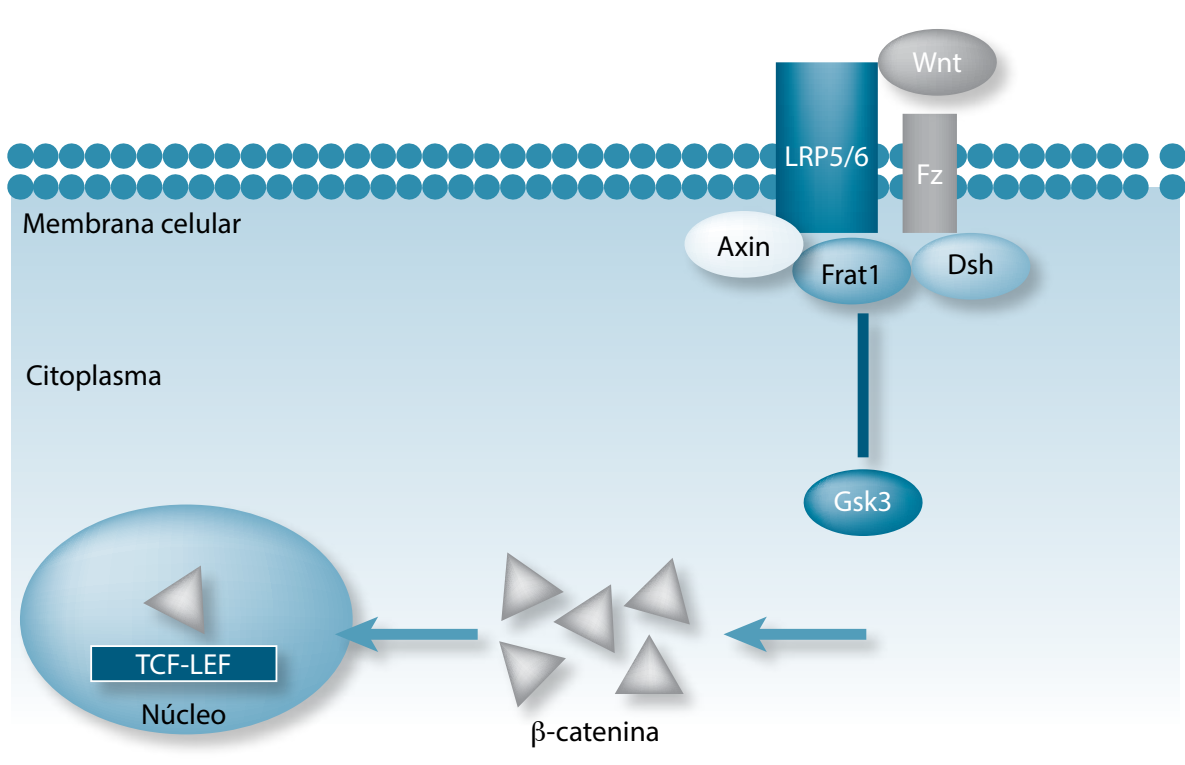

Modificado de J. Clin. Invest 2006;116:1202-120

Figura 3 .Vía de señalización Wnt / $\beta$-catenina: vía inactiva

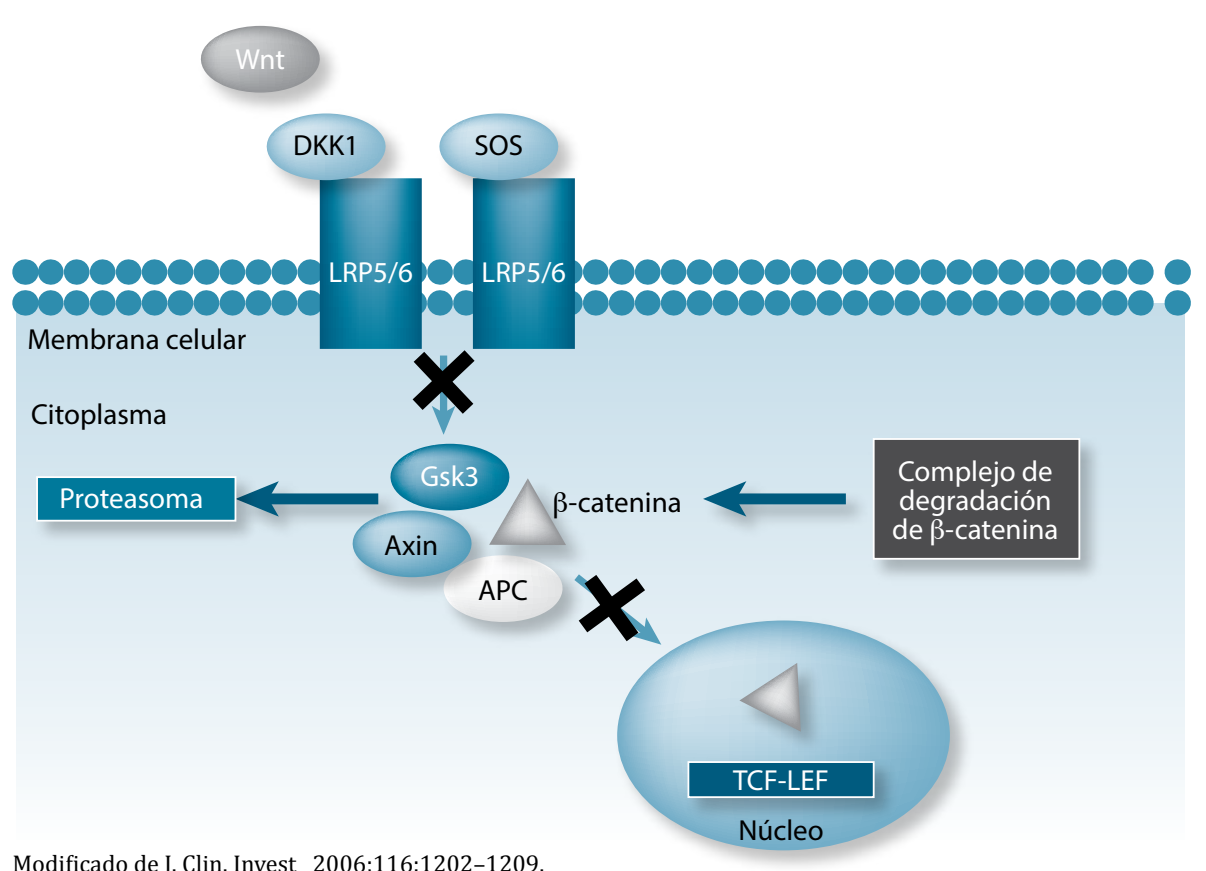

Modificado de J. Clin. Invest 2006;116:1202-1209.

ósea (figura 3). Las mutaciones que llevan a pérdida de la función del gen SOST están ligadas a enfermedades que se caracterizan por aumento de la masa ósea. Se describen dos fenotipos clínicos de estas mutaciones, la esclerosteosis y la enfermedad de Van Buchem, asociadas a fuerza esquelética inusual, masa ósea elevada y resistencia a fracturas ${ }^{(13,14) .}$
Estudios realizados en animales y humanos con anticuerpos antiesclerostina han demostrado aumentos marcados en la masa ósea, por tanto el bloqueo de la acción de la esclerostina con anticuerpos monoclonales es un nuevo enfoque terapéutico osteoanabólico prometedor para el tratamiento de la osteoporosis ${ }^{(14)}$.

\section{Anticuerpos antiesclerostina}

Moléculas en investigación y estudios existentes a la fecha:

Romosozumab (AMG 785): Anticuerpo (Ac) antiesclerostina monoclonal humanizado.

El primer estudio fue publicado en el año 2011, un ensayo clínico aleatorizado, doble ciego, fase 1 , de tres meses de seguimiento con 72 participantes, hombres sanos y mujeres posmenopáusicas. Recibieron una dosis única subcutánea o intravenosa de AMG 785 o placebo. Sus resultados fueron aumento de la DMO y marcadores de formación ósea y reducción (dosis dependiente) de marcadores de resorción ósea. Sus principales efectos adversos fueron eritema en el sitio de la aplicación, dolor lumbar, cefalea, estreñimiento, artralgias y mareos ${ }^{(15)}$.

El estudio más reciente fue publicado en enero de 2014, por Michael R. McClung et al. Es un estudio de fase 2 multicéntrico, aleatorizado, controlado con placebo, de grupos paralelos, cuyo objetivo fue evaluar la eficacia y seguridad de romosozumab durante un periodo de 12 meses en 419 mujeres posmenopáusicas con baja masa ósea. Las pacientes fueron aleatorizadas para recibir romosozumab mensual subcutáneo (dosis de 70 mg, 140 mg o 210 $\mathrm{mg}$ ) o cada tres meses (dosis de $140 \mathrm{mg}$ o $210 \mathrm{mg}$ ), o placebo o un comparador activo de forma abierta (alendronato 70 $\mathrm{mg} / \mathrm{semana}$ o teriparatida subcutánea $20 \mathrm{mcg} /$ día). Todas las dosis de romosozumab se asociaron con un aumento significativo en la densidad mineral ósea en la columna lumbar. Se reportó aumento en la DMO de $11,3 \%$ con la dosis mensual 
Tabla 1. Estudios fase $1 \mathrm{del}$ anticuerpo AMG 785

\begin{tabular}{|c|c|c|c|c|}
\hline $\begin{array}{l}\text { Clinicaltrials.gov } \\
\text { identifier }\end{array}$ & Diseño & Criterios de inclusión & Resultado primario & Dosis \\
\hline $\begin{array}{l}\text { NCT01101061 } \\
\text { Inicio: } 2010 \\
\text { Referencia: } 17\end{array}$ & $\begin{array}{l}\text { Doble ciego } \\
\text { Dosis única SC }\end{array}$ & $\begin{array}{l}\text { Mujeres posmenopáusicas } \\
\text { con osteopenia } \\
\text {-Japonesas ( } 45 \text { a } 70 \text { años) }\end{array}$ & Seguridad, tolerabilidad & $\begin{array}{l}\text { AMG } 785 \text { (Dosis: } 1 \mathrm{mg} / \\
\mathrm{kg}, 3 \mathrm{mg} / \mathrm{k} \text {, o } 5 \mathrm{mg} / \mathrm{kg} \text { ) vs. } \\
\text { placebo }\end{array}$ \\
\hline $\begin{array}{l}\text { NCT00950950 } \\
\text { Inicio: } 2009 \\
\text { Referencia: } 18\end{array}$ & $\begin{array}{l}\text { Doble ciego } \\
\text { Multidosis SC }\end{array}$ & $\begin{array}{l}\text { Mujeres sanas } \\
\text { posmenopáusicas ( } 55 \text { y } 80 \\
\text { años) con osteopenia }\end{array}$ & $\begin{array}{l}\text { Calidad de hueso del } \\
\text { antebrazo utilizando pQCT }\end{array}$ & $\begin{array}{l}\text { AMG785 } 3 \mathrm{mg} / \mathrm{kg} \text {, SC, cada } \\
\text { cuatro semanas durante } \\
\text { tres meses vs. placebo }\end{array}$ \\
\hline $\begin{array}{l}\text { NCT01833754 } \\
\text { Inicio: } 2013 \\
\text { Referencia: } 19\end{array}$ & $\begin{array}{l}\text { Etiqueta abierta } \\
\text { Dosis única }\end{array}$ & Falla renal estadio 4 y 5 & Seguridad & $\begin{array}{l}\text { Dosis única } \\
\text { (No reportado) }\end{array}$ \\
\hline $\begin{array}{l}\text { NCT01588509 } \\
\text { Inicio: } 2012 \\
\text { Referencia: } 20\end{array}$ & $\begin{array}{l}\text { Etiqueta abierta } \\
\text { Transición desde } \\
\text { Alendronato (1 año de } \\
\text { tratamiento) }\end{array}$ & $\begin{array}{l}\text { Mujeres posmenopáusicas } \\
\text { (55-85 años) T-score entre } \\
-2 \text { y -4 (CL o CT o CF) }\end{array}$ & $\begin{array}{l}\text { Porcentaje de cambio } \\
\text { de la DMO en columna } \\
\text { lumbar después de tres } \\
\text { meses de tratamiento }\end{array}$ & $\begin{array}{l}\text { Tres dosis } \\
\text { (No reportado) }\end{array}$ \\
\hline
\end{tabular}

(Publicados en la página electrónica: clinical trails.gov)

Tabla 2. Estudios fase 2 del anticuerpo AMG 785

\begin{tabular}{l|l|l|l}
\hline \multicolumn{1}{c|}{ Clinicaltrials.gov identifier } & \multicolumn{1}{|c|}{ Diseño } & \multicolumn{1}{c|}{ Criterios de inclusión } & \multicolumn{1}{c}{ Resultado primario } \\
$\begin{array}{l}\text { NCT00896532 } \\
\text { Inicio: } 2009 \\
\text { Referencia: } 21\end{array}$ & $\begin{array}{l}\text { Diseño doble ciego: } \\
\text { Comparado con placebo }\end{array}$ & $\begin{array}{l}\text { Mujeres posmenopáusicas: } \\
55-85 \text { años }\end{array}$ & $\begin{array}{l}\text { Variación \% respecto al valor } \\
\text { basal de DMO columna lumbar a } \\
\text { los } 12 \text { meses de tratamiento }\end{array}$ \\
& $\begin{array}{l}\text { Etiqueta abierta: alendronato y } \\
\text { teriparatide } \\
\text { Multidosis }\end{array}$ & $\begin{array}{l}\text { Densitometria T-score entre }-2,0 \\
\text { M-3,5 }\end{array}$ & \\
\hline
\end{tabular}

(Publicados en la página electrónica: clinical trails.gov)

Tabla 3. Estudios fase 3 del anticuerpo AMG 785

\begin{tabular}{|c|c|c|c|c|}
\hline $\begin{array}{l}\text { Clinicaltrials.gov } \\
\text { identifier }\end{array}$ & Diseño & Criterios de inclusión & Resultado primario & Dosis \\
\hline $\begin{array}{l}\text { NCT01796301 } \\
\text { Inicio: } 2013 \\
\text { Referencia: } 22\end{array}$ & $\begin{array}{l}\text { Etiqueta abierta } \\
\text { controlado con } \\
\text { teriparatide }\end{array}$ & $\begin{array}{l}\text { Mujeres posmenopáusicas, } \\
\text { dx osteoporosis ( } 60 \text { a } 90 \\
\text { años) } \\
\text { Tratamiento con } \\
\text { bifosfonatos orales } \\
\text { durante al menos tres } \\
\text { años inmediatamente } \\
\text { antes de la selección } \\
\text { Antecedente de fractura } \\
\text { por fragilidad vertebral o } \\
\text { no vertebral }\end{array}$ & $\begin{array}{l}\text { Porcentaje de cambio } \\
\text { en DMO en cadera total: } \\
\text { plazo } 12 \text { meses }\end{array}$ & $\begin{array}{l}\text { Tiempo de tratamiento } 12 \\
\text { meses } \\
\text { Dosis no reportadas }\end{array}$ \\
\hline $\begin{array}{l}\text { NCT01631214 } \\
\text { Inicio: } 2012 \\
\text { Referencia: } 23\end{array}$ & $\begin{array}{l}\text { Doble ciego controlado } \\
\text { con alendronato }\end{array}$ & $\begin{array}{l}\text { Mujeres posmenopáusicas } \\
\text { con osteoporosis y al } \\
\text { menos una fractura } \\
\text { vertebral, ( } 60 \text { a } 90 \text { años) }\end{array}$ & $\begin{array}{l}\text { Incidencia de nuevas } \\
\text { fracturas vertebrales: } \\
\text { Plazo: } 24 \text { meses }\end{array}$ & $\begin{array}{l}\text { Tiempo de tratamiento } 24 \\
\text { meses } \\
\text { Dosis no reportadas }\end{array}$ \\
\hline
\end{tabular}

(Publicados en la página electrónica: clinical trails.gov)

de $210 \mathrm{mg}$, en comparación con una disminución de $0,1 \%$ con placebo y aumentos de 4,1\% y 7,1\% con alendronato y teriparatida, respectivamente ${ }^{(16)}$.

Actualmente encontramos estudios fase 1, 2 y 3 del anticuerpo AMG 785, en diferentes etapas de desarrollo (publicados en la página electrónica: clinical trials.gov) que se resumen en las tablas 1, 2 y 3.
AMG 167: Anticuerpo antiesclerostina monoclonal AMG 167. Estudios en desarrollo fase 1, ver tabla 4.

Blosozumab: Anticuerpo antiesclerostina LY 2541546. Estudios fase 1 y 2 en desarrollo, ver tabla 5 .

BSP 804: Anticuerpo antiesclerostina BSP 804. Actualmente sólo un estudio fase 1 en desarrollo, ver tabla 6. 
Tabla 4: Estudios fase 1 del anticuerpo AMG 167

\begin{tabular}{|c|c|c|c|c|}
\hline $\begin{array}{l}\text { Clinicaltrials.gov } \\
\text { identifier }\end{array}$ & Diseño & Criterios de inclusión & Resultado primario & Dosis \\
\hline $\begin{array}{l}\text { NCT00902356 } \\
\text { Inicio: } 2009 \\
\text { Referencia: } 24\end{array}$ & $\begin{array}{l}\text { Doble ciego } \\
\text { Dosis única SC o IV }\end{array}$ & $\begin{array}{l}\text { Hombres sanos y mujeres } \\
\text { posmenopáusicas } \\
45 \text { y } 65 \text { años }\end{array}$ & Seguridad y tolerabilidad & $\begin{array}{l}5 \text { cohortes: única dosis de } \\
\text { AMG } 167 \text { vs. placebo } \\
\text { Mujeres posmenopáusicas } \\
\text { SC: } 21 \mathrm{mg}, 70,210,350 \text { o } \\
700 \mathrm{mg} \\
\text { IV: } 70 \mathrm{mg} \text { o } 350 \mathrm{mg} \text { IV } \\
\text { Hombres } \\
70 \mathrm{mg} \text { o } 350 \mathrm{mg} \mathrm{SC} \text { o IV }\end{array}$ \\
\hline $\begin{array}{l}\text { NCT01101048 } \\
\text { Inicio: } 2010 \\
\text { Referencia: } 25\end{array}$ & $\begin{array}{l}\text { Doble ciego } \\
\text { Dosis múltiples }\end{array}$ & $\begin{array}{l}\text { Hombres sanos y mujeres } \\
\text { posmenopáusicas con } \\
\text { osteopenia }\end{array}$ & Seguridad y tolerabilidad & $\begin{array}{l}7 \text { cohortes } \\
\text { Cohortes } 1 \text {-2 y 4: AMG } \\
\mathbf{1 6 7} \text { cada } 2 \text { semanas/total } \\
\text { de } 6 \text { dosis } \\
\text { Cohortes } 3-5 \text { y 6: AMG } \\
\mathbf{1 6 7} \text { cada } 4 \text { semanas/total } \\
\text { de } 3 \text { dosis } \\
\text { Cohortes } 7 \text { : AMG } \mathbf{1 6 7} \\
\text { cada } 2 \text { semanas/total de } \\
12 \text { dosis }\end{array}$ \\
\hline
\end{tabular}

(Publicados en la página electrónica: clinical trails.gov)

Tabla 5. Estudios fase 1 y 2 blosozumab

\begin{tabular}{|c|c|c|c|c|}
\hline $\begin{array}{l}\text { Clinicaltrials.gov } \\
\text { identifier }\end{array}$ & Diseño & Criterios de inclusión & Resultado primario & Dosis \\
\hline $\begin{array}{l}\text { NCT01742078 } \\
\text { Inicio: } 2008 \\
\text { Fase } 1 \\
\text { Referencia: } 26\end{array}$ & $\begin{array}{l}\text { Doble ciego } \\
\text { Dosis única }\end{array}$ & $\begin{array}{l}\text { Mujeres posmenopáusicas } \\
\text { sanas, entre } 45 \text { a } 70 \text { años }\end{array}$ & Seguridad, tolerabilidad & $\begin{array}{l}\text { Dosis IV: } 7,5 \mathrm{mg}, 25 \mathrm{mg}, 75 \\
\mathrm{mg}, 225 \mathrm{mg}, 750 \mathrm{mg} \\
\text { Dosis SC: } 150 \mathrm{mg}\end{array}$ \\
\hline $\begin{array}{l}\text { NCT01742091 } \\
\text { Inicio: } 2012 \\
\text { Fase } 1 \\
\text { Referencia: } 27\end{array}$ & Múltiples dosis & $\begin{array}{l}\text { Mujeres posmenopáusicas } \\
\text { sanas }\end{array}$ & Seguridad, tolerabilidad & $\begin{array}{l}\text { Ocho semanas de } \\
\text { tratamiento } \\
180 \mathrm{mg} \mathrm{SC} \mathrm{C/4} \mathrm{s} \\
270 \mathrm{mg} \mathrm{C} / 2 \mathrm{~s} \\
270 \mathrm{mg} \mathrm{SC} \mathrm{C/4} \mathrm{s} \\
540 \mathrm{mg} \text { (IV) C/4 s } \\
750 \mathrm{mg} \text { (IV) C/2 s }\end{array}$ \\
\hline $\begin{array}{l}\text { NCT01144377 } \\
\text { Inicio: } 2010 \\
\text { Fase } 2 \\
\text { Referencia: } 28\end{array}$ & $\begin{array}{l}\text { Doble ciego } \\
\text { Multidosis }\end{array}$ & $\begin{array}{l}\text { Mujeres posmenopáusicas } \\
\text { ( } 45-85 \text { años) } \\
\text { T-score, columna lumbar } \\
\text { entre }-2 \text { y }-3,5\end{array}$ & $\begin{array}{l}\text { Porcentaje de cambio en } \\
\text { la columna lumbar DMO: } \\
\text { plazo } 52 \text { semanas }\end{array}$ & $\begin{array}{l}180 \mathrm{mg} \mathrm{SC} \mathrm{C/4} \mathrm{s} \\
180 \mathrm{mg} \mathrm{C} / 2 \mathrm{~s} \\
270 \mathrm{mg} \mathrm{C} / 2 \mathrm{~s} \\
270 \mathrm{mg} \mathrm{C} / 12 \mathrm{~s}\end{array}$ \\
\hline
\end{tabular}

(Publicados en la página electrónica: clinical trails.gov)

Tabla 6 : Estudio fase 1 de BSP 804

\begin{tabular}{l|l|l|l|l}
\hline \multicolumn{1}{c|}{$\begin{array}{c}\text { Clinicaltrials.gov } \\
\text { identifier }\end{array}$} & \multicolumn{1}{|c|}{ Diseño } & \multicolumn{1}{c|}{ Criterios de inclusión } & \multicolumn{1}{c|}{ Resultado primario } & \multicolumn{1}{c}{ Dosis } \\
\hline $\begin{array}{l}\text { NCT01406548 } \\
\text { Inicio: } 2011\end{array}$ & $\begin{array}{l}\text { Doble ciego } \\
\text { Referencia: } 29\end{array}$ & $\begin{array}{l}\text { Mujeres posmenopáusicas, } \\
(45-85 \text { años })\end{array}$ & $\begin{array}{l}\text { Porcentaje de cambio } \\
\text { en DMO en la columna }\end{array}$ & No reportada \\
& & $\begin{array}{l}\text { T-score en columna } \\
\text { lumbar a los nueve meses }\end{array}$ & \\
& & lumbar entre $-2,0$ y $-3,5$ & $\begin{array}{l}\text { Eventos adversos, } \\
\text { Plazo nueve meses }\end{array}$ & \\
\hline
\end{tabular}

(Publicados en la página electrónica : Clinical trails.gov) 


\section{Proteína Dickkopf 1(DKK-1)}

Es una proteína que se expresa en osteoblastos y osteocitos, aunque también se ha demostrado su presencia por fuera del hueso en piel, placenta, próstata, plaquetas y endotelio ${ }^{(12)}$.

DKK-1 es un inhibidor de la vía Wnt y un regulador de la remodelación articular. En modelos animales de artritis reumatoide, el aumento de los niveles de DKK-1 está relacionado con mayor resorción ósea y disminución de su formación. Los corticoides y los procesos inflamatorios crónicos aumentan la expresión de DKK-1, inhibiendo la vía Wnt, explicando sus efectos deletéreos sobre el hueso ${ }^{(10)}$.

\section{Anticuerpos anti DKK-1}

Las terapias dirigidas contra DKK-1, con el objetivo de aumentar la masa ósea, ya están en desarrollo. En un modelo preclínico de artritis reumatoide el bloqueo de DKK-1 inhibió la pérdida ósea y en un modelo de mieloma múltiple la inhibición de DKK-1 impidió la formación de lesiones osteolíticas y aumentó la tasa de formación de hueso ${ }^{(30)}$. Actualmente se encuentra en desarrollo un estudio fase 1 de anticuerpos anti DKK-1 en hombres sanos y mujeres osteopénicas (ver tabla 7).

Posibles efectos adversos de la terapia con Ac antiesclerostina y anti DKK-1

La activación indiscriminada de Wnt podría resultar en mayor riesgo de desarrollo de tumores en tejidos no esqueléticos, como cáncer colorrectal, carcinoma hepatocelular entre otros tumores malignos. Soluciones potenciales incluyen la neutralización de los antagonistas Wnt de forma específica en las células óseas y su uso por periodos de tiempo limitados ${ }^{(2)}$.

\section{Superfamilia TGF- $\beta$.}

La superfamilia del factor de crecimiento transformante beta (TGF- $\beta$ ) está constituida por un grupo grande de proteínas multifuncionales. Estas proteínas afectan diversos procesos celulares que van desde la regulación de la diferenciación y proliferación celular hasta procesos fisiológicos complejos como la inflamación, la cicatrización de tejidos y la formación de hueso. Hacen parte de esta familia la activina y la BMP que juegan un papel clave en el proceso de formación ósea ${ }^{(32)}$.

\section{Señalización de BMP en la diferenciación de osteoblastos y la formación ósea}

Las proteínas BMP (BMP 2,4,5,6,7) son proteínas con capacidad osteogénica. El proceso de señalización se lleva a cabo por medio de la unión de BMP a su receptor de membrana tipo serina/treonina cinasa tipo I y tipo II (RI y RII), permitiendo la formación de un complejo heterodimérico que fosforila proteínas Smad (small mothers against decapentaplegic). Éstas, una vez activas, se dirigen al núcleo donde interactúan con el SBE (smad binding element), permitiendo una respuesta transcripcional que induce la expresión del gen Runx2 para controlar la diferenciación de células precursoras mesenquimales (figura 4) ${ }^{(33)}$.

Tabla 7. Estudio fase 1 de Ac anti DKK1: RN564

\begin{tabular}{|c|c|c|c|c|}
\hline $\begin{array}{l}\text { Clinicaltrials.gov } \\
\text { identifier }\end{array}$ & Diseño & Criterios de inclusión & Resultado primario & Dosis \\
\hline $\begin{array}{l}\text { NCT01293487 } \\
\text { Inicio: } 2011 \\
\text { Referencia: } 31\end{array}$ & $\begin{array}{l}\text { Doble ciego } \\
\text { Dosis única }\end{array}$ & $\begin{array}{l}\text { Hombre sanos y mujeres } \\
\text { con osteopenia entre } 55 \\
\text { años y } 80 \text { años }\end{array}$ & Seguridad, tolerabilidad & No reportadas \\
\hline
\end{tabular}

(Publicados en la página electrónica: clinical trails.gov)

Tabla 8. Estudio fase 1 de Rhbmp-2/CPM

\begin{tabular}{|c|c|c|c|c|}
\hline $\begin{array}{l}\text { Clinicaltrials.gov } \\
\text { identifier }\end{array}$ & Diseño & Criterios de inclusión & Resultado primario & Dosis \\
\hline $\begin{array}{l}\text { NCT00752557 } \\
\text { Inicio: } 2008 \\
\text { Referencia: } 34\end{array}$ & $\begin{array}{l}\text { Doble ciego } \\
\text { Comparador: } \\
\text { Bifosfonatos orales } \\
\text { Administración local en } \\
\text { cadera }\end{array}$ & $\begin{array}{l}\text { Mujeres posmenopáusicas, } \\
\text { entre } 65 \text { años y } 85 \text { años } \\
\text { Osteoporosis }\end{array}$ & $\begin{array}{l}\text { Porcentaje de cambios en } \\
\text { DMO, Plazo: } 12 \text { meses en } \\
\text { cadera }\end{array}$ & $\begin{array}{l}\text { Experimental: } 1 \mathrm{rhBMP}-2 \text { / } \\
\text { CPM, } 6 \mathrm{ml}: 1,0 \mathrm{mg} / \mathrm{ml} \\
\text { Bifosfonatos orales } \\
\text { (risedronato, alendronato } \\
\text { o ibandronato sódico) } \\
\text { Experimental. } 2 \mathrm{rhBMP}-2 \text { / } \\
\text { CPM, } 6 \mathrm{ml}: 2,0 \mathrm{mg} / \mathrm{ml} \\
\text { Bifosfonatos } \\
\text { Comparador activo: } 3 \\
\text { Intervención: bifosfonatos }\end{array}$ \\
\hline
\end{tabular}

(Publicados en la página electrónica: clinical trails.gov) 


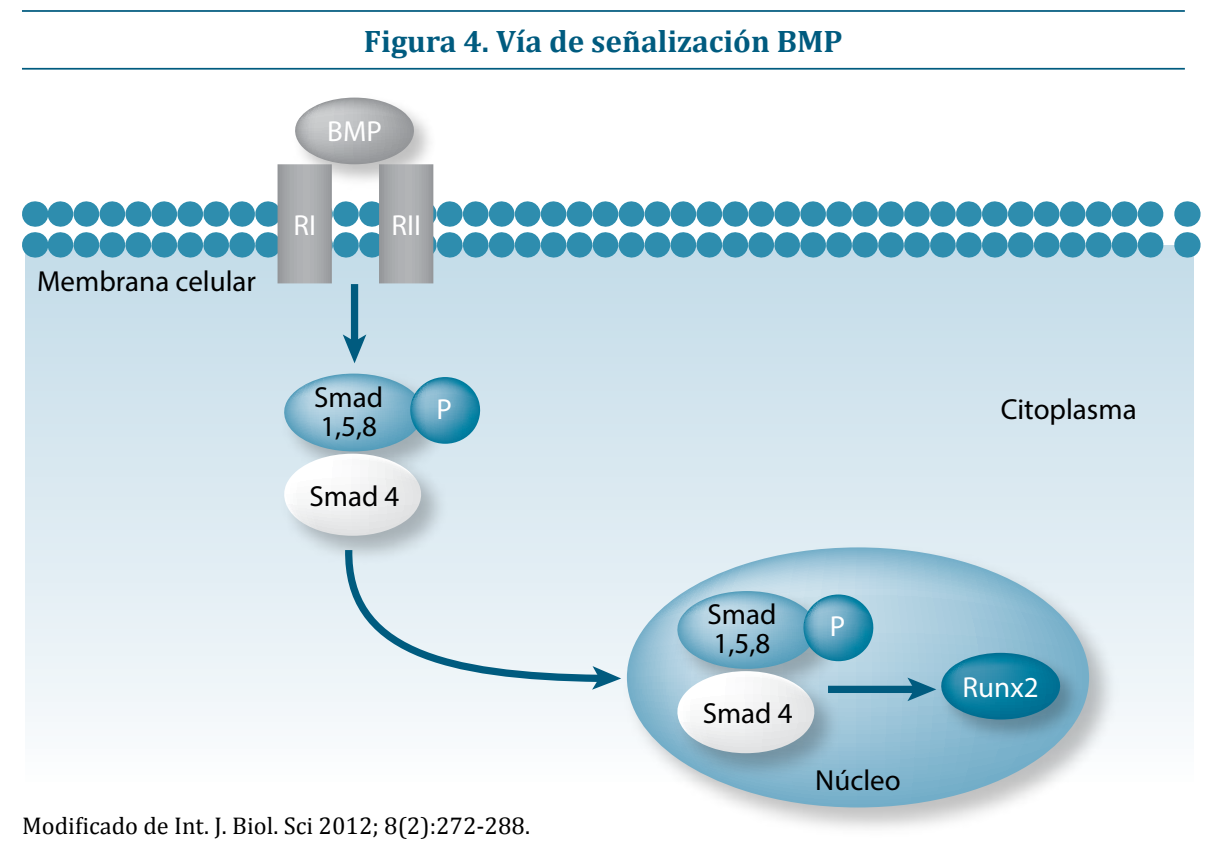

\section{Utilidad potencial de las BMPs como terapia anabólica en osteoporosis}

Se han realizado estudios con rhBMP-2 (recombinant human BMP-2) en la curación de fracturas, retardo en la curación de fracturas y pseudoartrosis, tanto en ratas como en humanos. Los estudios realizados en ratas osteoporóticas para el tratamiento local de fracturas demostraron un rápido aumento de la resistencia ósea en la zona fracturada y aceleración de la consolidación ósea. Los efectos adversos reportados en estudios ortopédicos con el uso local fueron osificación heterotópica, reacciones inmunes menores, edema e infecciones. La administración sistémica puede asociarse con anomalías graves en el desarrollo y función celular ${ }^{(1)}$. Actualmente se encuentra en curso un estudio fase 1 del uso de Rhbmp-2/CPM en mujeres osteoporóticas con el objetivo de evaluar el porcentaje de cambio en la DMO en un plazo de 12 meses (ver tabla 8).

\section{Conclusión}

La mejor comprensión de los reguladores moleculares del metabolismo óseo y de sus vías moduladoras ha llevado a la investigación y desarrollo de nuevos agentes terapéuticos con efecto anabólico óseo, entre estos los anticuerpos monoclonales anti-DKK-1 y antiesclerostina (de uso sistémico) y la proteína morfogénica del hueso 2 recombinante humana (rhBMP-2) para uso local.

Los tratamientos de primera línea contra la osteoporosis son antirresortivos. Éstos, aunque han demostrado evidencia en la prevención de fracturas, carecen de propiedades que mejoren la microarquitectura ósea.

Si bien teriparatida es una terapia formadora de hueso y ha demostrado aumentar el número de trabéculas, conexiones intertrabeculares, el grosor cortical y la geometría del hueso, las nuevas terapias anabólicas, a diferencia de teriparatida, son netamente formadoras y no tienen ningún efecto resortivo, esto podría tener un impacto en cuanto a ganancia de masa ósea y calidad de hueso. Sin embargo, se necesitan más estudios para evaluar su seguridad a largo plazo, ya que podría haber un mayor riesgo de tumores en tejidos no esqueléticos.

Contamos actualmente con estudios de nuevos medicamentos en diferentes fases de desarrollo (fase 1, 2 y 3) que demuestran beneficios adicionales a las terapias actualmente disponibles, y que se perfilan como una prometedora alternativa a mediano y largo plazo en el manejo de la osteoporosis.

\section{Referencias}

1. Konstantinos A. Toulis, Athanasios D. Anastasilakis, Stergios A. Polyzos, Polyzois Makras. Targeting the osteoblast: approved and experimental anabolic agents for the treatment of osteoporosis. HORMONES 2011;10(3):174-195.
2. Canalis E. Update in New Anabolic Therapies for Osteoporosis. J Clin Endocrinol Metab 2010; 95: 1496-1504.

3. Baron R, Hesse E. Update on Bone Anabolics in Osteoporosis Treatment: Rationale, Current Status, and Perspectives. J Clin Endocrinol Metab 2012;97: 311-325. 
4. Lippuner k. The future of osteoporosis treatment a research update. Swiss Med Wkly 2012;142:w13624.

5. Canalis E, Giustina A, Bilezikian J. Mechanisms of Anabolic Therapies for Osteoporosis. N Engl J Med 2007;357:905-16.

6. Lewiecki E Michael. Monoclonal antibodies for the treatment of osteoporosis. Expert Opin. Biol. Ther 2013;13(2):183-196.

7. Krishnan V, Bryant H,1 MacDougald O. Regulation of bone mass by Wnt signaling. J. Clin. Invest 2006;116:1202-1209.

8. Pierre J. Marie. Transcription factors controlling osteoblastogenesis. Archives of Biochemistry and Biophysics 2008; 473:98-105.

9. Piters E, Boudin E, Van Hul W. Wnt signaling: A win for bone. Archives of Biochemistry and Biophysics 2008;473:112-116.

10. Daoussis D, Andonopoulos A. The Emerging Role of Dickkopf-1 in Bone Biology: Is It the Main Switch Controlling Bone and Joint Remodeling?. Semin Arthritis Rheum 2011;41:170-177.

11. Veverka V, Henry A, Slocombe P, Ventom A, Mulloy B, Muskett F, et al. Characterization of the Structural Features and Interactions of Sclerostin. J. Biol. Chem 2009;284:10890-10900.

12. Hua Zhu Ke, Richards W, Li X, Ominsky M. Sclerostin and Dickkopf-1 as Therapeutic Targets in Bone Diseases. Endocrine Reviews 2012;33(5):747-783.

13. Lewiecki E Michael. Sclerostin monoclonal antibody therapy with AMG 785: a potential treatment for osteoporosis. Expert Opin. Biol. Ther 2011; 11(1):117-127.

14. Costa A, Bilezikian J. Sclerostin: Therapeutic Horizons Based Upon Its Actions. Curr Osteoporos Rep 2012;10:64-72.

15. Padhi D, Jang G, Stouch B, Fang L, Posvar E. Single-Dose, Placebo-Controlled, Randomized Study of AMG 785, a Sclerostin Monoclonal Antibody. Journal of Bone and Mineral Research 2011; 26(1):19-26.

16. McClung M, Grauer A, Boonen S, Bolognese M, Brown J, Diez-Perez A, et al. Romosozumab in Postmenopausal Women with Low Bone Mineral Density. N Engl J Med 2014;370:412-20.

17. Clinicaltrials.gov. Number, NCT01101061. A Single-dose Study Evaluating AMG 785 in Healthy Postmenopausal Japanese Women.

18. Clinicaltrials.gov. Number, NCT00950950. A Study to Evaluate the Effect of AMG 785 on Bone Quality of the Forearm in Postmenopausal Women With Low Bone Mass.

19. Clinicaltrials.gov. Number, NCT01833754. Study of Romo- sozumab (AMG 785) Administered to Healthy Subjects and Subjects With Stage 4 Renal Impairment or Stage 5 Renal Impairment Requiring Hemodialysis.

20. Clinicaltrials.gov. Number, NCT01588509. Transition From Alendronate to AMG 785.

21. Clinicaltrials.gov. Number, NCT00896532. Phase 2 Study of AMG 785 in Postmenopausal Women With Low Bone Mineral Density.

22. Clinicaltrials.gov. Number, NCT01796301. An Open-label Study to Evaluate the Effect of Treatment With AMG 785 or Teriparatide in Postmenopausal Women (STRUCTURE).

23. Clinicaltrials.gov. Number, NCT01631214. Study to Determine the Efficacy and Safety of Romosozumab in the Treatment of Postmenopausal Women With Osteoporosis.

24. Clinicaltrials.gov. Number, NCT00902356. A First-in-human Study Evaluating AMG 167 in Healthy Men and Postmenopausal Women.

25. Clinicaltrials.gov. Number, NCT01101048. An Ascending Multiple Dose Study Evaluating AMG 167 in Healthy Men and Postmenopausal Women With Low Bone Mineral Density.

26. Clinicaltrials.gov. Number, NCT01742078. A Study of LY2541546 in Healthy Postmenopausal Women.

27. Clinicaltrials.gov. Number, NCT01742091. A Multiple Dose Study of LY2541546 in Healthy Postmenopausal Women.

28. Clinicaltrials.gov. Number, NCT01144377. A Study of LY2541546 in Women With Low Bone Mineral Density.

29. Clinicaltrials.gov. Number, NCT01406548. Safety and Efficacy of Multiple Dosing Regimens of BPS804 in Post Menopausal Women With Low Bone Mineral Density.

30. Rachner T, Khosla S, Hofbauer L. Osteoporosis: now and the future. Lancet 2011; 377: 1276-87.

31. Clinicaltrials.gov. Number, NCT01293487. Safety And Tolerability Study Of RN564 In Women With Osteopenia And Healthy Men.

32. Sosa-Garrocho M, Macías-Silva $\mathrm{M}$. El factor de crecimiento transformante beta (TGF-b): funciones y vías de transducción. REB 2004;23 (1): 3-11.

33. Chen G, Deng C, Li Yi-Ping. TGF- $\beta$ and BMP Signaling in Osteoblast Differentiation and Bone Formation. Int. J. Biol. Sci 2012; 8(2):272-288.

34. Clinicaltrials.gov. Number, NCT 00752557. Study Evaluating Changes In Bone Mineral Density (BMD), And Safety Of Rhbmp-2/CPM In Subjects With Decreased BMD. 